\title{
Importancia de los glucósidos cianogénicos en el sabor de frutos de almendros (Prunus dulcis Miller) y su incidencia en la agroindustria
}

\section{Importance of cyanogenic glycosides in the flavor of almond fruits (Prunus dulcis Miller) and their impact on agribusiness}

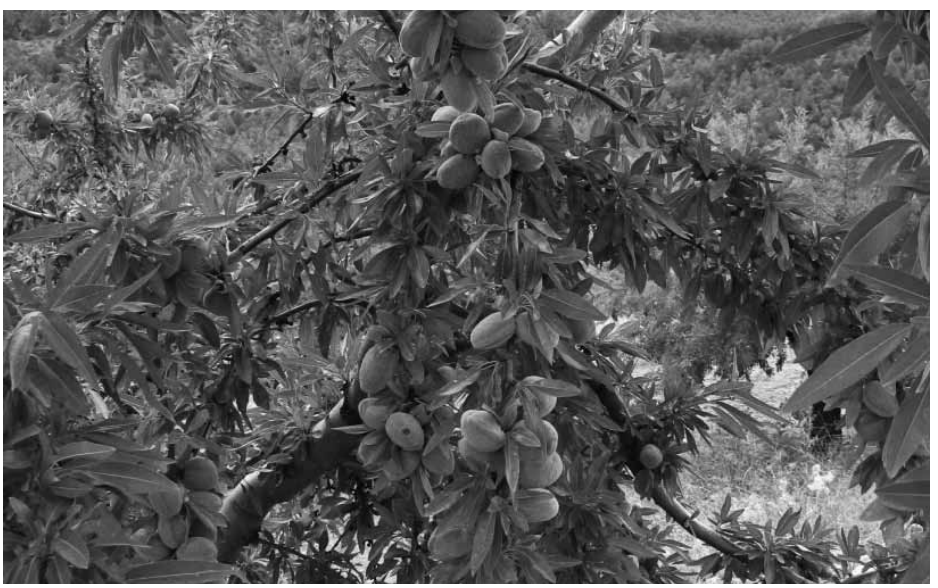

GUILLERMO ARRÁZZOLA ${ }^{1,4}$

NURIA GRANÉ 2

FEDERICO DICENTA ${ }^{3}$

Árbol de almendra

en fructificación.

Foto: F. Dicenta

\section{RESUMEN}

Durante el proceso de acondicionamiento de la almendra para su consumo o como materia prima en la obtención de derivados se encuentran almendras amargas, situación que representa un problema cuando no se necesitan, esto debido a la degradación de la prunasina y amigdalina en benzaldehído y ácido cianhídrico, lo cual influye en el sabor y aroma de la almendra destinada para usos industriales. El objetivo de la investigación fue clasificar 29 variedades de almendras atendiendo la concentración de amigdalina y prunasina en los tres subgrupos de manera a priori (dulce, ligeramente amargo y amargo) para su posible utilización en la agroindustria. Se distinguieron dos grandes grupos, amargas y no amargas, mediante cromatografía líquida de alta resolución (HPLC) con contenidos promedios entre 2.400 hasta 5.900 mg de amigdalina/100 g muestra, para amargas. Para el grupo de las no amargas como 'Primorskii', 'Ramillete' y 'Bonita', la amigdalina no se detectó, otras presentaron valores de $20 \mathrm{mg}$ amigdalina/100 g. Algunas variedades no amargas presentaron en el primer año (2008) valores ligeramente menores que durante el segundo año (2009), por ejemplo las variedades Achaak, Carretas, Ferraduel (10,22 a 11,25 mg de amigdalina/100 g). El estudio permitió clasificar las variedades en amargas y no amargas, lo cual posibilitará mejorar y disminuir los problemas de sabor en la industria (turrones, mazapán, licor) donde las almendras se utilizan sin amargor.

\footnotetext{
Facultad de Ingeniería, Programa Ingeniería de Alimentos, Universidad de Córdoba, Montería (Colombia).

2 Facultad de Ciencias, Departamento de Química Analítica, Nutrición y Ciencia de los Alimentos, Universidad de Alicante, Alicante (España).

3 Departamento de Mejora Vegetal, Centro de Edafología y Biología Aplicada del Segura Consejo Superior de Investigaciones Científicas (CEBAS-CSIC), Murcia (España).

4 Autor para correspondencia.guillermo.arrazola@ua.es
} 


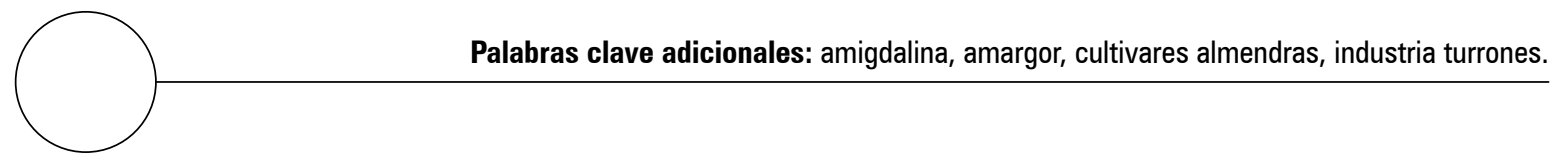

\section{ABSTRACT}

During the conditioning process of kernels for consumption or as raw material for the preparation of derivatives, bitter almonds are present, which create problems when they are not required because the degradation of prunasin and amygdalin into benzaldehyde and hydrogen cyanide influences the taste and aroma of almonds destined for industrial use. The research objective was to classify 29 varieties of almonds based on amygdalin concentration into three previously established subgroups (sweet, slightly sour and bitter) for possible use in agribusiness. Only two groups of kernels, bitter and non-bitter, were distinguished by high pressure liquid chromatography (HPLC), with an average content between 2,400 and 5,900 $\mathrm{mg}$ of amygdalin/100 g of sample for bitter types. For the non-bitter group, such as 'Primorskii', 'Ramillete' and 'Bonita', amygdalin was not detected, but others had values of $20 \mathrm{mg}$ amygdalin/100 g. Some non-bitter varieties presented (2008) values in the first year that were slightly lower than in the second year (2009), for example Achaak, Carretas, and Ferraduel (10.22 to $11.25 \mathrm{mg}$ of amygdalin/100 g). This study allowed for the classification of the varieties into bitter and non-bitter, which will improve and reduce the problems of taste in the industry (nougat, marzipan liquor) where non-bitter almonds are used.

Additional key words: amygdalin, bitterness, almond cultivars, nougat industry.

El almendro (Prunus dulcis Miller) es una especie con una gran variabilidad genética por lo cual hay gran cantidad de variedades. Durante la recolección de las cosechas del almendro frecuentemente se encuentran almendras amargas, producto de variedades que poseen este sabor debido a la presencia de glucósidos cianogénicos amigdalina y prunasina (McCarty y Leslie, 1952; Conn, 1980; Polesello y Rizzolo, 1989; Frehner et al., 1990). Estos compuestos, tienen la capacidad de producir ácido cianhídrico en condiciones muy determinadas de $\mathrm{pH}$ y en presencia de enzimas específicas (Vetter, 1999).

Dada la variabilidad en el sabor durante los programas de mejora genética, los descendientes amargos son eliminados durante el proceso de selección, para su uso industrial, lo cual reduce la eficacia del programa de mejora, especialmente en la obtención de variedades autocompatibles, donde además permite potencializar los sabores para su uso agroindustrial (Arrázola et al., 2002). Los beneficios de las almendras para la salud de las personas son múltiples, ya que contienen: agua, proteínas, grasas, hidratos de carbono, los ocho aminoácidos esenciales y celulosa; vitaminas $\mathrm{B}_{1}, \mathrm{~B}_{2}, \mathrm{C}, \mathrm{A}, \mathrm{D}$ y E; calcio, fósforo, hierro, potasio, sodio, magnesio, azufre, cloro, manganeso, cobre y zinc; constituyendo un alimento imprescindible en una dieta sana y equilibrada (Agunbiade y Olanlokun, 2006). Dado su alto contenido en fibra se utilizan como laxante y antiinflamatorio del aparato digestivo y urinario. Además, la almendra es uno de los frutos 
secos con mayor cantidad de vitamina E por lo que ejerce un valioso papel antioxidante. Posee un $52 \%$ de grasas, de las cuales las dos terceras partes corresponden al ácido oleico, por lo cual, comer almendras es muy parecido a tomar aceite de oliva desde el punto de vista cardiovascular (Hernández y Zacconi, 2009). Otro punto a tener en cuenta, es el contenido de ácido linoleico (omega-6), ácido graso que no sintetiza el organismo y es esencial por lo cual le es necesario obtener de la dieta (Agunbiade y Olanlokun, 2006). Existe otra variedad botánica de almendras, llamada Prunus amygdalus var. Amara, que es la que produce almendras amargas; estas son tóxicas para el organismo, por lo que no se deben consumir (Agunbiade y Olanlokun, 2006). A diferencia de las dulces, poseen en su interior una sustancia llamada amigdalina (Laetril o vitamina B-17). Cuando se parte o mastica una de estas almendras se pone en contacto dicha sustancia con la saliva y la emulsina; esta última es una enzima $\beta$-glucosidasa que actúa fraccionando la amigdalina en $\beta$-D-glucosa (hidrato de carbono), benzaldehído (responsable del sabor amargo) y ácido cianhídrico, debido al sabor desagradable del aldehído, se evita la ingesta del ácido cianhídrico, el cual es el responsable del envenenamiento, siendo la dosis mortal equivalente a 20 almendras para los adultos y 10 para los niños (Hernández y Zacconi, 2009). Muchas variedades cultivadas son heterocigóticas para este carácter, y aunque algunas de ellas son ligeramente amargas, la mayoría son dulces (Pérez et al., 2012). Un caso extremo en este sentido han sido los programas de mejora en Estados Unidos que han utilizado el amargo Prunus webbii como genitor autocompatible, lo que ha generado hasta el $50 \%$ de las descendencias amargas cuando el otro genitor (Carmel, Livingstone), fue heterocigótico. Aunque en general se acepta que las variedades ligeramente amargas son heterocigóticas para este carácter, actualmente la única manera de saber si una variedad es homocigótica o heterocigótica es estudiando a sus descendientes, tarea que es enormemente laboriosa, costosa y que requiere varios años de estudio, autores como (Surleva y
Drochioiu, 2013) han modificado el método de detección de cianógenos totales en almendras, cuyos resultados se tienen en cuenta para determinar el ácido cianhídrico que se produce a partir de la Linamarina en Manihot esculenta, muy utilizada en países tropicales para consumo humano (raíz) y animal (raíz, hojas y tallos) como suplemento. Yildirim y Askin (2010) evaluaron la variabilidad de otros Prunus como el albaricoque, también muy utilizados en la industria de zumos, néctares y almíbares. Algunos autores han apuntado que el sabor ligeramente amargo posiblemente sea en cierto modo independiente del carácter dulce o amargo y en consecuencia está determinando otros genes, que podrían actuar modificando cuantitativamente la producción de benzaldehído (Dicenta, 1991; Dicenta y García, 1993). Pérez et al. (2008), estudiando cruzamientos entre variedades heterocigóticas, observaron que los descendientes ligeramente amargos fueron más numerosos cuando el genitor heterocigótico fue ligeramente amargo. Si los homocigóticos dulces y los heterocigóticos tuvieran diferentes contenidos de amigdalina, podrían reconocerse a través de un análisis químico simple. Esto permitiría diseñar los cruzamientos evitando la aparición de descendientes amargos, o bien asumiendo a priori el riesgo de obtener estos amargos, si el cruzamiento fuera especialmente interesante por otras circunstancias. La clasificación de cultivares teniendo en cuenta el contenido de amigdalina y prunasina en almendras maduras, influye en productos de mejor calidad que tienen como materia prima almendras, donde el sabor amargo incide en la aceptación o rechazo, además industrias de derivados de jugos de frutas tropicales, como papaya, maracuyá, aceites de uso farmacéutico (almendras), licores derivados de almendras, cuyos productos estén libres de amargor, evitarían ser rechazados (Arrázola, 2002). Miller y Tuck (2013) determinaron concentraciones de 23 a $1.263 \mathrm{~g}$ HCN/100 g muestra, hoy por hoy consumidores de almendras enteras presentan quejas debido al sabor amargo encontrado en muchos productos del mercado (Toomey et al., 2012). 
El objetivo de este trabajo fue determinar el contenido de compuestos cianogénicos en relación con el sabor de la semilla de diferentes variedades y estimar las posibilidades de usos agroindustriales de almendras y derivados controlando el sabor final del producto procesado o en fresco.

\section{MATERIALES Y MÉTODOS}

\section{Material vegetal}

Se utilizaron 29 variedades de almendra procedentes de la colección del Centro de Edafología y Biología Aplicada del Segura (CEBAS), Murcia (España) en los años 2008 (año 1) y 2009 (año 2). Dado que en la colección no se disponía de variedades amargas, se incluyeron seis descendientes amargos de Garrigues x Tuono. La mayoría son de genotipo conocido, tras el estudio de sus descendencias (Dicenta, 1991).

- DD (dulces siempre): Del Cid, Ferragnès, Peraleja, Primorskii, Ramillete y Titán.

- Dd (dulces, a veces ligeramente amargas): Marcona, Desmayo Largueta, Atocha.

- Dd (ligeramente amargas): Garrigues, Genco, Tuono.

- dd (amargas siempre): S3060, S3062, S3076, S3108, S3112, S3126.

- El resto de muestras fueron de sabor dulce pero de genotipo desconocido (DD o Dd): Achaak, Bonita, Carretas, CEBAS, Colorada, Ferraduel, La Mona, Pajarera, Planeta, Rumbeta y Tioga.

Entre las variedades dulces, Desmayo Largueta, Atocha y La Mona tienen un ligerísimo (casi inapreciable) sabor amargo. Las muestras de almendra fueron recogidas en estado de madurez comercial, obteniendo solamente la almendra libre de la cáscara, molida, acondicionada y preparadas para su posterior análisis, mediante liofilización utilizando Liofilizador Telstar 2000 (Cataluña, España), a una presión de $4 \times 10^{-2}$ mbar y una temperatura entre $-79^{\circ} \mathrm{C} y-82^{\circ} \mathrm{C}$, con el fin de que no se descongelaran.

\section{Extracción y determinación de los glucósidos cianogénicos por HPLC}

Se pesaron 0,2 g de almendra si era amarga y 0,4 $\mathrm{g}$ si la almendra era dulce o ligeramente amarga. Se introdujo en un tubo de centrífuga junto con $5 \mathrm{~mL}$ de metanol de grado HPLC, se tapó y se dejó durante 12 h en baño con agitación a $30^{\circ} \mathrm{C}$. Se centrifugó durante 5 min a 3.000 rpm se dejó reposar tratando de no mezclar nuevamente el sedimento formado. El sobrenadante se tomó con una jeringa apropiada filtrándola a través de un disco Millipore de Nylon de 0,45 $\mu \mathrm{m}$, para eliminar las impurezas que pudieran afectar en la resolución del cromatograma y acortar la vida útil de la columna cromatográfica. Cuando la muestra obtenida era de almendras dulces ligeramente amargas se inyecta el extracto filtrado directamente, pero en el caso de las amargas dependiendo de la concentración del analito en la muestra se diluyó 1:5, 1:10 o incluso 1:20.

\section{Condiciones cromatográficas}

Se utilizó un equipo de cromatografía líquida de alta eficiencia marca Waters Modelo 600 Controller, entrega de solventes con gradiente cuaternario modelo 600E, inyector manual Rheodine 7125, detector de arreglo de diodos Waters modelo 996, columna Symmetry C18, Waters, $250 \times 4,6 \mathrm{~mm}, 5 \mu \mathrm{m}$ ), además una precolumna C18 con objeto de preservar la columna, software Millenium 2008. Otras condiciones cromatográficas fueron: volumen de muestra $20 \mu \mathrm{L}$, flujo del eluyente 1,3 $\mathrm{mL} \mathrm{min}^{-1}$, composición del eluyente: acetonitrilo: agua (20:80) isocrática, tiempo de elución: $10 \mathrm{~min}$, análisis mediante el método patrón externos, curva de calibrado amigdalina y prunasina. 


\section{Análisis estadístico}

Se analizaron los resultados de los datos promedios (triplicado) del contenido de amigdalina (mg/100 g de muestra seca) del conjunto de variedades durante los dos años (2008 y 2009) de estudio, bajo un diseño completamente al azar. Mediante un análisis de varianza se determinaron diferencias en los contenidos de compuestos cianogénicos presentes, amigdalina, entre años y variedades, realizando posteriormente una comparación de medias (Tukey). Se tuvieron un total de 174 unidades experimentales. Este mismo análisis se repitió considerando únicamente las variedades dulces y ligeramente amargas, utilizando software estadístico SPSS 21.0 (Statistical Package for the Social Sciences).

\section{RESULTADOS Y DISCUSIÓN}

La tabla 1 muestra los resultados del análisis de varianza del contenido de amigdalina de las almendras de las variedades estudiadas durante dos años. Los resultados obtenidos ponen de manifiesto la existencia de diferencias estadísticamente significativas respecto al contenido de amigdalina entre las distintas variedades y entre los dos años. También fue significativa la interacción entre variedad y el año. A pesar de las diferencias estadísticas, las almendras recolectadas en (año 1) tuvieron un contenido medio de amigdalina (4.325 mg/100 g) casi igual al de las recolectadas en el año 2 (4.344 mg/100 g). El análisis anual de los resultados indica que algunas variedades presentaron en el año 1 valores ligeramente menores (como Achaak, Colorada, Carretas, Ferraduel, S3076, S3060) o ligeramente mayores (como Desmayo Largueta, Genco, S3126, S3062). Sin embargo, la mayoría de las variedades mantuvieron valores de amigdalina parecidos en los dos años, como en el caso de Ramillete, Bonita, Marcona etc. (figura 1 y 2). Tanto en el análisis anual (figura 1 y 2) como en el global (figura 3), se observa que todas las muestras dulces y ligeramente amargas forman un subconjunto homogéneo, cuyos valores medios de contenido de amigdalina no se diferencian estadísticamente entre sí. Las muestras amargas se diferenciaron claramente del resto, aunque entre ellas se pueden distinguir cuatro grupos distintos. Los resultados muestran una enorme diferencia del contenido de amigdalina en las variedades estudiadas. El contenido medio de amigdalina en las variedades amargas fue mucho mayor (hasta 1.000 veces) que el de las "no amargas" (dulces y ligeramente amargas). Se presentó homogeneidad del grupo de las no amargas, mientras que en algunas variedades como Primorskii, Ramillete y Bonita la amigdalina no fue detectada, en otras como Ferraduel, Garrigues, Tuono y Pajarera se alcanzaron concentraciones superiores a $20 \mathrm{mg} / 100$ g. El resto de las variedades presentaron va-

Tabla 1. Análisis de varianza para contenido de amigdalina entre variedades y años (2008 y 2009), para la totalidad de las variedades estudiadas.

\begin{tabular}{|l|r|r|r|r|r|}
\hline Fuente variación & Suma de cuadrados & Grados de libertad & Cuadrado medio & F. ratio & Significancia F \\
\hline Efectos principales & 571.790 .282 & 23 & 19.716 .906 & 18.779 .41 & $<0,001$ \\
\hline Año & 9.359 & 1 & 9.364 & 8.52 & 0,005 \\
\hline Variedad & 571.786 .323 & 28 & 20.420 .990 & 19.447 .95 & $<0,001$ \\
\hline Año x variedad & 107.438 & 22 & 4.881 & 4.45 & $<0,001$ \\
\hline Explicada & 571.897 .730 & 52 & 11.213 .680 & 10.670 .00 & $<0,001$ \\
\hline Residual & 112.243 & 105 & 1.040 & & \\
\hline Total & 572.010 .063 & 156 & 3.620 .315 & & \\
\hline
\end{tabular}




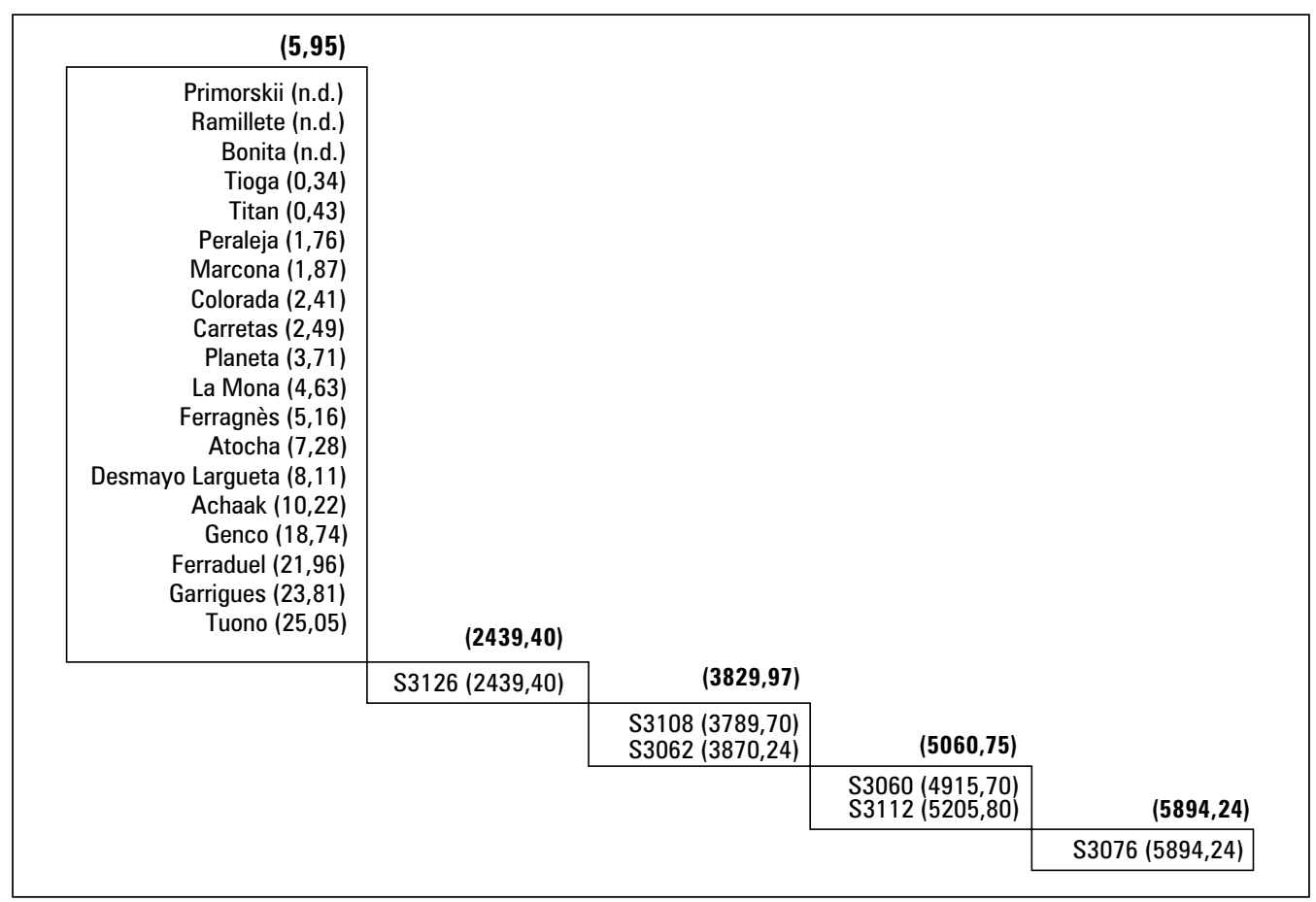

Figura 1. Subconjuntos de varianza de amigdalina $(\mathrm{mg} / 100 \mathrm{~g})$ para la totalidad de las variedades, año 2008. Entre paréntesis el contenido medio por variedad y en la parte superior el del respectivo subconjunto. Límite de detección $=0,387 \mathrm{mg} / 100 \mathrm{~g}$ de muestra seca.

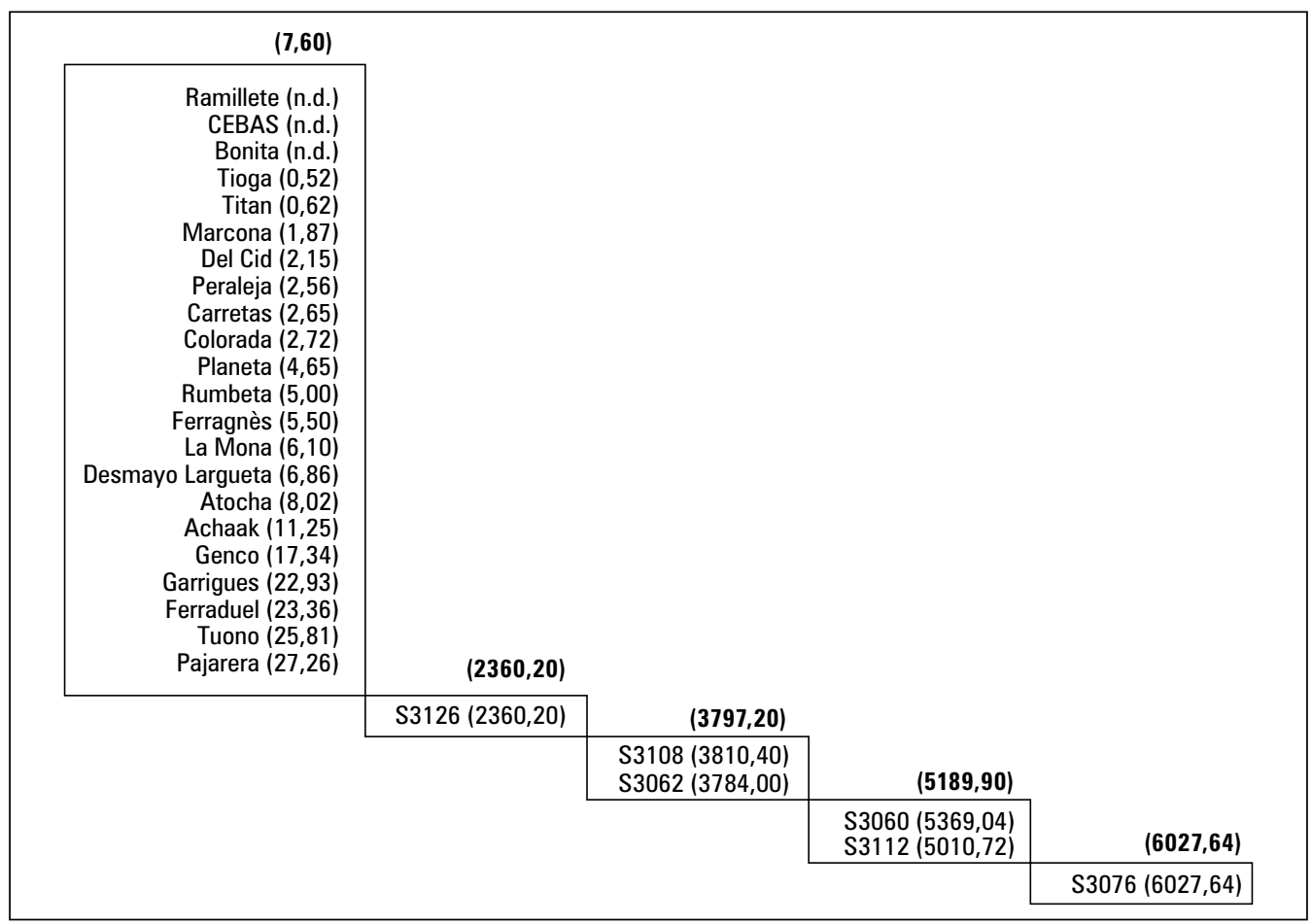

Figura 2. Subconjuntos de varianza de amigdalina $(\mathrm{mg} / 100 \mathrm{~g})$ para la totalidad de las variedades, año 2009. Entre paréntesis el contenido medio de cada variedad y en la parte superior de cada casilla el del respectivo subconjunto. Límite de detección $=0,387 \mathrm{mg} / 100 \mathrm{~g}$ de muestra seca. 


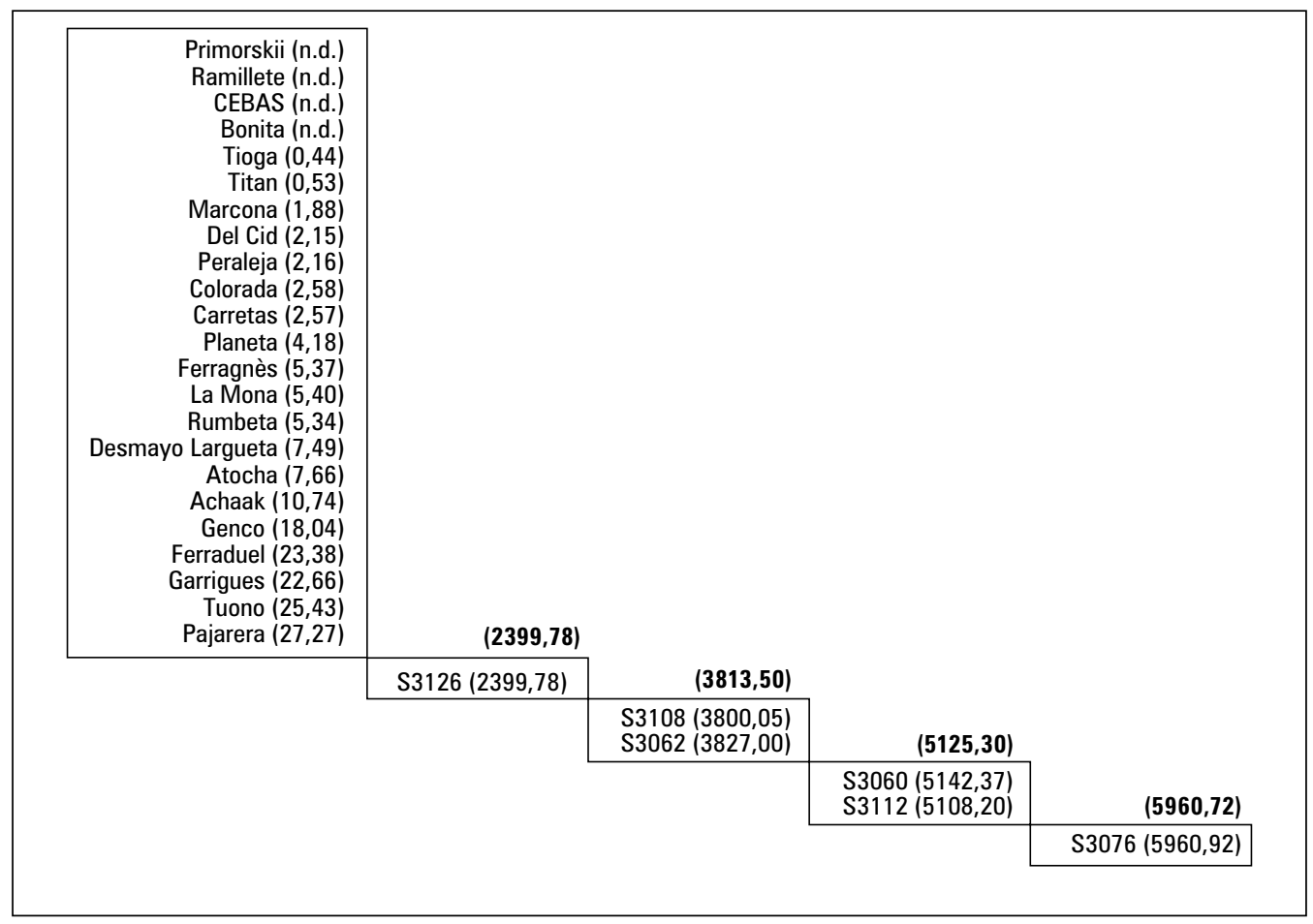

Figura 3. Subconjuntos de varianza de amigdalina $(\mathrm{mg} / 100 \mathrm{~g})$ para la totalidad de las variedades estudiadas en años 2008 y 2009, conjuntamente. Entre paréntesis el contenido medio de cada variedad y en la parte superior de cada casilla el del respectivo subconjunto. nd = no detectado. Límite de detección $=0,387 \mathrm{mg} / 100 \mathrm{~g}$ de muestra seca.

lores intermedios, que se distribuyen de una manera continua dentro de este intervalo. Los individuos amargos presentaron valores entre $2.400 \mathrm{mg} / 100 \mathrm{~g}$ (S3126) y casi $6.000 \mathrm{mg} / 100 \mathrm{~g}$ (S3076). Así, la concentración de amigdalina no permite discriminar, en este caso, entre variedades dulces y ligeramente amargas, solamente se puede diferenciar entre amargas y "no amargas". Esta última diferenciación no es de una gran utilidad práctica, para la industria de bebidas, mazapán, crema de leche almendra y turrones, ya que esta clasificación también puede hacerse sencillamente probando las almendras, donde para uso agroindiustrial es importante determinar los niveles mínimos que puedan causar amargor al fruto.

El contenido de compuestos cianogénicos ha sido estudiado en diferentes plantas. Chassagne et al. (1996), estudiando semillas de variedades de maracuyá, obtuvieron concentraciones de 31 mg kg-1 de muestra seca. Shahidi y Wanasundara (1997) encontraron concentraciones de 7.000 $\mathrm{mg} / 100 \mathrm{~g}$ de peso fresco de linamarina en lima y de $500 \mathrm{mg} / 100 \mathrm{~g}$ de peso fresco de taxipilina en bambú. Además, en semillas de lino detectaron linamarina (300 mg/100 g muestra seca) y linustatina $(6.000 \mathrm{mg} / 100 \mathrm{~g}$ de muestra seca).

También hay algunos estudios del contenido de amigdalina en semillas de frutales de hueso. Lucas y Sotelo (1984) determinaron el contenido de ácido cianhídrico (obtenido a partir de la hidrólisis de la amigdalina) en semillas de diferentes Prunus, como ciruelo (233 mg/100 g), albaricoquero (136 mg/100 g), cerezo (131 mg/100 g) y manzano (42 mg/100 g). En semillas de albaricoquero, Femenia et al. (1995) obtuvieron concentraciones de amigdalina entre $4.400 \mathrm{y}$ $6.500 \mathrm{mg} / 100 \mathrm{~g}$ de muestra seca y Gómez et al. (1998) entre 5.500 y $7.000 \mathrm{mg} / 100 \mathrm{~g}$ de muestra seca. Otros investigadores como Stoewsand et 
al. (1975), Briggs y Yuen (1978), Mandenius et al. (1983) y Voldrich et al. (1989) encontraron igualmente altos contenidos de amigdalina en semillas amargas de albaricoque.

En el caso de la almendra, Corradi y Micheli (1982) determinaron concentraciones de 3.300 mg de ácido cianhídrico/kg de muestra seca. Usai y D'hallewin (1992) determinaron concentraciones de $9.255 \mathrm{mg}$ de amigdalina/kg muestra seca para la variedad amarga Sassari 11 y $1.056 \mathrm{mg} /$ $\mathrm{kg}$ de muestra seca en la variedad dulce Arrubia. McCarty y Leslie (1952) estudiando semillas de melocotonero y almendras, e híbridos de almendro x melocotonero, analizaron el benzaldehído obtenido por hidrólisis enzimática de la amigdalina, obteniendo porcentajes entre 0,241 y $0,619 \%$ en melocotoneros, entre 0,001 y $0,007 \%$ en almendrasy 0,004 y $0,444 \%$ en los híbridos almendro x melocotonero. Los valores encontrados por estos autores fueron mucho mayores que los resultados obtenidos en este trabajo en el caso de las variedades Genco (390 frente $18 \mathrm{mg} / 100$ g) y Tuono (750 frente $25 \mathrm{mg} / 100 \mathrm{~g}$ ). Polesello y Rizzolo (1989) señalaron un contenido de 2,5 a $3,5 \%$ de amigdalina en almendras amargas. En general, las diferentes concentraciones de amigdalina, encontradas en semillas amargas de diferentes Prunus, están dentro del rango determinado, entre 2.400 y $6.000 \mathrm{mg} / 100 \mathrm{~g}$ en almendras amargas, contrario a los resultados por Barbera et al. (1987) y Usai D'hallewin (1992), quienes encontraron valores muy bajos para almendras amargas y muy altas para las dulces.

Dadas las enormes diferencias observadas respecto al contenido de amigdalina entre las variedades amargas y no amargas, y al objeto de poder distinguir entre ligeramente amargas $y$ dulces en función de su contenido en amigdalina, se analizó este contenido. La tabla 2 muestra los resultados del análisis de varianza entre variedades y años para las variedades "no amargas". Al igual que en el estudio conjunto de todas las variedades, los resultados aquí obtenidos muestran la existencia de diferencias estadísticamente significativas respecto al contenido de amigdalina entre las distintas variedades y entre los dos años. También fue significativa la interacción entre variedad y año. La prueba de Tukey indica la presencia de diferentes subconjuntos homogéneos para los datos anuales y para el estudio conjunto de los dos años, en general las variedades dulces tienen menos amigdalina que las ligeramente amargas. Si bien todas las variedades ligeramente amargas tienen niveles elevados de amigdalina (respecto a los niveles de las almendras no amargas), no todas las variedades que presentan estos niveles fueron ligeramente amargas, este es el caso de, 'Pajarera' y 'Ferraduel'. Por lo tanto, mientras que en las almendras ligeramente amargas la

Tabla 2. Análisis de la varianza para contenido de amigdalina entre variedades y años (2008 y 2009), para las variedades dulces y ligeramente amargas.

\begin{tabular}{|l|r|r|r|r|r|}
\hline Fuente de variación & Suma cuadrados & Grados libertad & Cuadrado medio & F. ratio & Significancia F \\
\hline Efectos principales & 9196.735 & 23 & 399.857 & 4.761 .248 & $<0.001$ \\
\hline Año & 1.930 & 1 & 1.930 & 22.985 & $<0.001$ \\
\hline Variedad & 9176.641 & 22 & 417.121 & 4.966 .791 & $<0.001$ \\
\hline Año x variedad & 16.713 & 18 & 0.983 & 11.707 & $<0.001$ \\
\hline Explicada & 9213.448 & 40 & 230.336 & 2.742 .693 & $<0.001$ \\
\hline Residual & 6.887 & 84 & 0.084 & & \\
\hline Total & 9220.334 & 123 & 75.577 & & \\
\hline
\end{tabular}


concentración de amigdalina varió de 18 a 25 $\mathrm{mg} / 100 \mathrm{~g}$, en las almendras dulces varió desde "no detectable" a $27 \mathrm{mg} / 100 \mathrm{~g}$.

Lo anterior parece indicar que no hay una relación estrecha entre el sabor ligeramente amargo $y$ el contenido de amigdalina, y que para que una variedad sea ligeramente amarga tiene que tener algo de amigdalina, pero además se deben dar otros factores (probablemente bioquímicos), ausentes en el caso de Ferraduel y Pajarera (Arrázola et al., 2012). Así, la determinación de los individuos ligeramente amargos, ligada a la capacidad humana de detectar bajas cantidades de amigdalina, podría estar influenciada por la concentración de otros compuestos en la almendra, ácidos grasos presentes, taninos, flavonoides entre otros. Por otro lado, los resultados obtenidos por Dicenta y García (1993) ponen de manifiesto que las variedades ligeramente amargas (Garrigues) tienden a producir más descendientes ligeramente amargos que otras variedades dulces. Estas variedades amargas podrían trasmitir a sus descendencias la capacidad de producir estas sustancias modificadoras del sabor. Entre las variedades Achaak, Ferraduel y Pajarera quedan integradas en subconjuntos formados por las variedades ligeramente amargas $\mathrm{Dd}$, mientras que el resto de variedades de genotipo desconocido se integran dentro de los subconjuntos formados por variedades de genotipo amargo. La variedad de genotipo conocido DD que presenta el valor medio de contenido de amigdalina más alto y por lo tanto menos diferenciado de las variedades de genotipo Dd es la variedad Ferragnès. Muy probablemente, variedades como Bonita o CEBAS podrían ser dulces.

\section{CONCLUSIONES}

Se determinaron diferentes concentraciones de amigdalina entre las variedades estudiadas, donde se encontró una variación en el contenido de compuestos cianogénicos respecto al tiempo (año 1 y año 2), además el contenido de amigdalina de la almendra es característico de cada variedad. Las almendras amargas pueden distinguirse de las no amargas (dulces y ligeramente amargas) por su elevado contenido de amigdalina, amargas $(5.960,72 \mathrm{mg} / 100 \mathrm{~g}$ muestra), las almendras ligeramente amargas no pueden diferenciarse de las dulces en función del contenido de amigdalina, aunque parece que todas las ligeramente amargas, de 0,00 a 1,87 mg/100 g de muestra, tienen cierta cantidad de amigdalina. Los resultados obtenidos permiten identificar con certeza el sabor y aroma de una variedad dada en función de su concentración del contenido del compuesto(s) cianogénicos presente(s) con la posibilidad de disminuir y controlar las alteraciones por sabor amargo de productos terminados a partir de almendras como materia prima o para su consumo en fresco.

\section{REFERENCIAS BIBLIOGRÁFICAS}

Agunbiade, S. y J. Olanlokun. 2006. Evaluation of some nutritional characteristics of Indian almond (Prunus amigdalus) nut. Pakistan J. Nutr. 5(4), 316- 318.

Arrázola, G. 2002. Análisis de glucósidos cianogénicos en variedades de almendro: implicaciones en la mejora genética. Tesis de doctorado. Universidad de Alicante, Alicante, España.

Arrázola, G., R. Sánchez, R. Dicenta y N. Grané. 2012. Content of the cyanogenic glucoside amygdalin in almond seeds related to the bitterness genotype. Agron. Colomb. 30(2), 260-265.

Barbera, G., L. Di Marco, G. Fatta Del Bosco y P. Inglese. 1987. Behaviour of 26 almond cultivars growing under rainfed and semiarid conditions in Sicily. pp. 17-32. En: Memorias 7. Colloque du Grempa, Reus, España.

Briggs, D. y D. Yuen. 1978. Determination of cyanide in apricot kernels. Proc. Nutr. Soc. Aust. 3, 103-104. 
Chassagne, D., J.C. Crouzet, L.C. Bayonove y R.L. Baumes. 1996. Identification and quantification of passion fruit cyanogenic glycosides. J. Agric. Food Chem. 44, 3817-3820.

Conn, E.E. 1980. Cyanogenic compound. Annu. Rev. Plant Physiol. 31, 433-451.

Corradi, C. y G. Micheli. 1982. Total hydrocyanic acid content of macaroons. Industry Alimentary 21(1), $459-466$

Dicenta, F. 1991. Mejora genética del almendro por cruzamientos intervarietales: Herencia de caracteres y selección. Tesis de doctorado. Universidad de Murcia, Murcia, España.

Dicenta, F. y J.E. García. 1993. Inheritance of the kernel flavour in almond. Heredity 70, 308-312.

Femenia, A., C. Rosello, A. Mulet y J. Canellas. 1995. Chemical composition of bitter and sweet apricot kernel. J. Agric. Food Chem. 43, 356-361.

Frehner, M., M. Scalet y E.E. Conn. 1990. Pattern of the cyanide-potential in developing fruits. Plant Physiol. 94, 28-34.

Gómez, E., L. Burgos, C. Soriano y J. Marín. 1998. Amygdalin content in the seeds of several apricot cultivars. J. Sci. Food Agric. 77, 184-186.

Hernández, S. y F. Zacconi. 2009. Aceite de almendras dulces: Extracción, caracterización y aplicación. Quimica Nova 32(5), 1342-1345

Lucas, B. y A. Sotelo. 1984. A simplified test for the quantitation of cyanogenic glucoside in wild and cultivated seeds. Nutr. Rep. Intl. 29(3), 711-719.

Mandenius, C., L. Buelov y B. Danielsson. 1983. Determination of amygdalin and cyanide in industrial food samples using enzymic methods. Acta Chem. Scand. 8, 739-742.

McCarty, C.D. y J.W. Leslie. 1952. Frost HB.Bitterness of kernels of almond $\mathrm{x}$ peach hybrids and their parents. Proc. Amer. Soc. Hort. Sci. 59, 254-258.

Miller, R. y K. Tuck. 2013. Reports on the distribution of aromatic cyanogenic glycosides in Australian tropical rainforest tree species of the Lauraceae and Sapindaceae. Phytochem. 92, 146-152.

Pérez, R., K. Jørgensen, C.E. Olsen, F. Dicenta y B.L. Møller. 2008. Bitterness in almonds. Plant Physiol. 146, 1040-1052

Pérez, R., G. Arrázola, M.L. Martín, N. Grané y F. Dicenta. 2012. Influence of the pollinizer in the amygdalin content of almonds. Sci. Hortic. 139(18), 62-65.

Polesello, A. y A. Rizzolo. 1989. Caratteristiche nutrizionali e utilizzazione industrial delle mandorle. Frutticoltura 51(4), 43-50.

Shahidi, F. y P. Wanasundara P. 1997. Cyanogenic glycosides of flaxseeds. Amer. Chem. Soc. 10, 171-185.

Stoewsand, G., J.L. Anderson y R. Lamb. 1975. Cyanide content of apricot kernels. J. Food Sci. 40, 11071120 .

Surleva, A. y G. Drochioiu. 2013. A modified ninhydrin micro-assay for determination of total cyanogens in plants. Food Chem. 141, 2788-2794.

Toomey, V., E. Nuckum y C. Flurer. 2012. Cyanide and amygdalin as indicators of the presence of bitter almonds in imported raw almonds. J. Forensic Sci. 57(5), 1313-1317.

Usai, M. y G. D'hallewin. 1992. Cyanogenic glucosides contained in different organs of bitter and sweet almond. pp. 1233-1236. Agriculture rapport. 14081 FR. Amélioration génétique de deuxespèces de fruits secs méditerranéens: l'amandieret le pistachier. Memorias VIII Grempa Meeting, 26-27 June, Nîmes, Francia.

Vetter, J. 1999. Plant cyanogenic glycosides. Toxicon. $38,11-36$

Voldrich, M., H. Opatova, V. Kyzling y J. Hrdlicka. 1989. Determination of hydrogen cyanide and cyanogenic glycosides in fresh and preserved fruits. Czech J. Food Sci. 7(4), 271-278.

Yildirim, F. y A. Askin. 2010. Variability of amygdalin content in seeds of sweet and bitter apricot cultivars in Turkey. Afr. J. Biotechnol. 9(39), 6522-6524 